\title{
An empirical evaluation of the impact of missing data on treatment effect
}

\author{
Royes Joseph ${ }^{*}$, Julius Sim, Reuben Ogollah, Martyn Lewis \\ From 3rd International Clinical Trials Methodology Conference \\ Glasgow, UK. 16-17 November 2015
}

\section{Objectives}

Missing data represent a potential source of bias in randomized clinical trials (RCTs). A simple approach that makes use of the responses subsequently obtained via reminder is proposed to assess the validity of the inferences from a missing at random (MAR)-based primary analysis of incomplete RCTs.

\section{Methods}

We explored mechanism behind the reminder responses in two pragmatic RCTs - the TATE and STarT Back trials - by utilizing the fact that data that are recovered through reminders would otherwise have been missing. The present approach considered two data scenarios: (i) with the actual dataset and (ii) with a modified dataset, where outcome responses obtained after a certain number of reminders were treated as missing. The impact of the reminder responses was assessed by comparing the estimates from MAR-based analyses between the two data scenarios.

\section{Results}

In the TATE trial, the reminder approach showed that an MAR-based analysis was likely to yield biased estimates of treatment effect. Therefore, further sensitivity analyses were required under a range of plausible missing not at random (MNAR) assumptions. However, in the STarT Back trial, this approach showed that an MAR-based analysis was likely to yield an unbiased estimate of treatment effect.

\section{Conclusion}

The proposed reminder approach can be used to assess the robustness of the MAR assumption by checking expected consistency in MAR-based estimates. If the

\footnotetext{
Research Institute for Primary Care and Health Sciences, Keele University,
} Keele, Staffordshire, UK

(c) 2015 Joseph et al. This is an Open Access article distributed under the terms of the Creative Commons Attribution License (http:// creativecommons.org/licenses/by/4.0), which permits unrestricted use, distribution, and reproduction in any medium, provided the original work is properly cited. The Creative Commons Public Domain Dedication waiver (http://creativecommons.org/publicdomain/ zero/1.0/) applies to the data made available in this article, unless otherwise stated. 